This item was submitted to Loughborough's Research Repository by the author.

Items in Figshare are protected by copyright, with all rights reserved, unless otherwise indicated.

\title{
Evidence for polaronic Fermi liquid in manganites
}

PLEASE CITE THE PUBLISHED VERSION

PUBLISHER

(C) American Physical Society

LICENCE

CC BY-NC-ND 4.0

\section{REPOSITORY RECORD}

Alexandrov, A.S., Guo-Meng Zhao, H. Keller, B. Lorenz, Y.S. Wang, and C.W. Chu. 2019. "Evidence for Polaronic Fermi Liquid in Manganites". figshare. https://hdl.handle.net/2134/1320. 


\title{
Evidence for polaronic Fermi liquid in manganites
}

\author{
A. S. Alexandrov, ${ }^{1}$ Guo-meng Zhao, ${ }^{2}$ H. Keller, ${ }^{2}$ B. Lorenz, ${ }^{3}$ Y. S. Wang, ${ }^{3}$ and C. W. $\mathrm{Chu}^{3,4}$ \\ ${ }^{1}$ Department of Physics, Loughborough University, Loughborough LE11 3TU, United Kingdom \\ ${ }^{2}$ Physik-Institut der Universiät Zürich, CH-8057 Zürich, Switzerland \\ ${ }^{3}$ Department of Physics and Texas Center for Superconductivity, University of Houston, Houston, Texas 77204 \\ ${ }^{4}$ Lawrence Berkeley National Laboratory, 1 Cyclotron Road, Berkeley, California 94720
}

(Received 1 June 2001; published 17 September 2001)

\begin{abstract}
The modern multipolaron theory predicts a polaronic Fermi-liquid state, but such a state has not been unambigously confirmed by experiment so far. Here we report theoretical and experimental studies of the isotope effects on the low temperature kinetic properties of the doped ferromagnetic manganites. Our results provide clear evidence for a polaronic Fermi-liquid state in doped ferromagnetic manganites.
\end{abstract}

DOI: $10.1103 /$ PhysRevB.64.140404

PACS number(s): 75.30.Vn, 71.38.Cn, 74.20.Mn

Over the past decades, very interesting phenomena such as high-temperature superconductivity and colossal magnetoresistance have been discovered in several doped oxides. These doped oxides are characterized by significant carrier densities $\left(\geqslant 10^{21} \mathrm{~cm}^{-3}\right)$ and low mobility of the order or even less than the Mott-Ioffe-Regel limit $\left(e a^{2} / \hbar\right.$ $\sim 1 \mathrm{~cm}^{2} / \mathrm{Vs}$, where $a$ is the lattice constant) at room temperature. The very nature of the low-temperature "metallic" state of these materials can be hardly understood within the framework of the canonical theory of metals. However, it has been known for many decades starting from the pioneering work by Landau ${ }^{1}$ that the local lattice deformation can transform electrons into "self-trapped" polarons in doped ionic insulators. Further extensive theoretical studies (for review, see Refs. 2 and 3) have shown that polarons behave like heavy particles, and can be mobile with metallic conduction at sufficiently low temperatures. Under certain conditions ${ }^{4}$ they form a polaronic Fermi liquid with some properties being very different from ordinary metals. One of them is the effective mass of polarons. It is well known that electrons can change their mass in solids due to the interactions with ions, spins, and themselves. The renormalized (effective) mass of electrons is independent of the ion mass $M$ in ordinary metals where the Migdal adiabatic approximation is believed to be valid. However, if the interactions between electrons and nuclear ions are strong and/or the adiabatic approximation is not applied electrons form polarons (quasiparticles dressed by lattice distortions), so their effective mass $m^{*}$ will depend on $M$. This is because the polaron mass $m^{*}=m \exp (A / \omega),{ }^{5}$ where $m$ is the band mass in the absence of the electron-phonon interaction, $A$ is a constant, and $\omega$ is a characteristic phonon frequency which depends on the masses of ions. Hence, there is a large isotope effect on the carrier mass in polaronic metals, in contrast to the zero isotope effect in ordinary metals.

The total exponent of the isotope effect on $m^{*}$ is defined as $\alpha_{m *}=\Sigma-d \ln m^{*} / d \ln M_{i}\left(M_{i}\right.$ is the mass of the $i$ th atom in a unit cell). From this definition and the expression for the polaron mass $m^{*}$ mentioned above, one readily finds

$$
\alpha_{m} *=-\frac{1}{2} \ln \left(m^{*} / m\right)
$$

Interestingly, the same isotope exponent is predicted for the intersite bound pairs of polarons (i.e., bipolarons). ${ }^{5,6}$ The underlying assumption used to obtain this equation is the harmonic approximation for phonons and the inverse coupling perturbation expansion based on the Lang-Firsov canonical transformation, which is valid in a wide region of the coupling with high-frequency optical phonons. ${ }^{4}$

A large isotope effect on the effective mass of supercarriers has been observed in the high-temperature cuprate superconductors, ${ }^{7-9}$ which suggests that polarons and/or bipolarons should be involved in high-temperature superconductivity. Furthermore, in doped manganites, the polaronic nature of the carriers in the high-temperature paramagnetic insulating state has been shown by several independent experimental results such as the sign anomaly of the Hall effect, $^{10}$ the Arrhenius behavior of the drift and Hall mobilities, ${ }^{10}$ the giant oxygen-isotope shift of the Curie temperature $T_{C},{ }^{11}$ and the electron paramagnetic resonance linewidth which is proportional to the conductivity. ${ }^{12}$

On the other hand, the very nature of the charge carriers in the low-temperature metallic state of doped manganites has not been understood. In a theory of colossal magnetoresistance for ferromagnetic manganites ${ }^{13}$ polarons are considered as the carriers even in the low-temperature metallic state, while others ${ }^{14}$ believe that polaronic effects are not important at low temperatures. Some experimental studies ${ }^{15}$ concluded that polaronic effects may be involved in the ferromagnetic metallic state of the low $T_{C}$ compounds, while many others explained their unusual behavior in terms of a percolative conduction mechanism. ${ }^{16}$

Theoretically it has been demonstrated that the coherent motion of small polarons can lead to metallic conduction at low temperatures. ${ }^{2,17,4}$ In order to provide decisive evidence for the "metallic" polaronic liquid in doped ferromagnetic manganites, it is essential to study the isotope effects on the low-temperature kinetic and thermodynamic properties. These properties are dominated by the impurity and electronelectron scatterings, so the only source of the isotope effect might be the polaron mass dependence on the ion mass. For example, replacing ${ }^{16} \mathrm{O}$ with ${ }^{18} \mathrm{O}$ will increase the polaron mass, leading to the changes in the residual resistivity, and in other kinetic and thermodynamic properties. Here we provide clear evidence for the polaronic liquid in doped ferro- 


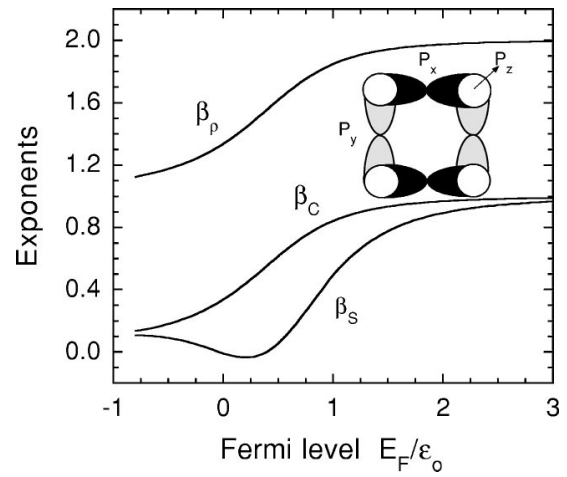

FIG. 1. The numerical results for three exponents: $\beta_{\rho}, \beta_{C}$, and $\beta_{S}$ as a function of the Fermi level $\mu \equiv E_{F} / \epsilon_{0}$. Inset: The oxygen orbitals of the cubic perovskite lattice.

magnetic manganites at low temperatures from theoretical and experimental studies of the isotope effects on kinetic and thermodynamic properties.

We first define and calculate three exponents: $\beta_{C}$ $=d \ln C_{\mathrm{el}} / d \ln m^{*}, \quad \beta_{\rho}=d \ln \rho_{o} / d \ln m^{*}, \quad$ and $\quad \beta_{S}$ $=d \ln S / d \ln m^{*}$ in the polaronic liquid. Here $C_{\mathrm{el}}$ is the electronic specific heat at low temperatures, $\rho_{o}$ is the residual resistivity, and $S$ is the low-temperature thermopower. We assume that the Fermi level (chemical potential) lies above the mobility edge, so polarons propagate in the Bloch states as heavy fermions ${ }^{17,4}$ scattered by the short-range impurity potential. The polaronic band structure might be quite complicated in manganites involving a few different orbital states of oxygen and manganise and electron-electron correlation effects. While our further consideration is largely independent on the band structure details, we choose a simple model of the polaron spectrum supported by the site selective experiments. Several experiments ${ }^{18,19}$ have shown that doped holes in manganites mainly reside on the oxygen orbitals for the doping level less than 0.4. Within a tight binding approximation, the oxygen hole energy spectrum consists of three bands formed by the overlap of $p_{x}, p_{y}$, and $p_{z}$ orbitals, respectively (see the schematic picture in the inset of Fig. 1). These bands are quasi-one-dimensional (1D) due to a large anisotropy in the hopping integrals [i.e., $t_{p p \pi}=-(1 / 4) t_{p p \sigma}^{2}$ ]. The polaronic mass enhancement is normally larger along the directions with a much smaller hopping integral. ${ }^{20}$ This will increase the anisotropy further so that the polaron bands become practically 1D. Three different bands are not mixed because the off-diagonal hopping integrals (e.g., $t_{p_{x}, p_{y}^{\prime}}$ ) are zero for the nearest neighbors. The random potential does not mix them either if it varies smoothly on the atomic scale. Therefore, there are three degenerate 1D-like polaronic oxygen hole bands in a cubic perovskite compound. Such a model allows us to derive the isotope exponents analytically. The polaronic propagator, $G(k, E)=\left[E-k^{2} / 2 m^{*}\right.$ $-\Sigma(k, E)]^{-1}$ is found with the self-energy, which is momentum $(k)$-independent in the noncrossing ("ladder") approximation,

$$
\Sigma(E) \propto \sum_{k} G(k, E) .
$$

Taking the integral in Eq. (2) one obtains the algebraic cubic equation for $\Sigma(E)$ with a solution:

$$
\begin{aligned}
\sigma(\epsilon)= & \frac{\epsilon}{3}-\left(\frac{1-i 3^{1 / 2}}{2}\right)\left[\frac{1}{16}+\frac{\epsilon^{3}}{27}+\left(\frac{1}{256}+\frac{\epsilon^{3}}{216}\right)^{1 / 2}\right]^{1 / 3} \\
& -\left(\frac{1+i 3^{1 / 2}}{2}\right)\left[\frac{1}{16}+\frac{\epsilon^{3}}{27}-\left(\frac{1}{256}+\frac{\epsilon^{3}}{216}\right)^{1 / 2}\right]^{1 / 3} .
\end{aligned}
$$

Here we introduce the dimensionless energy, $\epsilon=E / \epsilon_{0}$, and the self-energy $\sigma(\epsilon)=\Sigma(E) / \epsilon_{0}$ using the scattering rate $\epsilon_{0}$ $=\left(D^{2} m^{*} / \hbar^{2}\right)^{1 / 3}$ as the energy unit. The constant $D$ is the second moment of the Gaussian white-noise potential, $D$ $=2\left(n_{\mathrm{im}} v^{2} / a^{2}\right)$, where $n_{\mathrm{im}}$ is the impurity density, and $v$ is the coefficient of the $\delta$-function impurity potential. This selfenergy is almost exact above the mobility edge as shown with the exact solution for a one-dimensional particle in a random white-noise potential. ${ }^{21}$

The specific heat is proportional to the density of states (DOS) at the (dimensionless) Fermi level, $\mu=E_{F} / \epsilon_{0}$. Assuming three degenerate spin-polarized oxygen bands, we find

$$
\frac{C_{\mathrm{el}}}{T}=\frac{2 \pi k_{B}^{2}}{\hbar a^{2}}\left(\frac{m^{*}}{\epsilon_{0}}\right)^{1 / 2} g(\mu)
$$

with $g(\epsilon)=\operatorname{Im} \sigma(\epsilon)$. The residual conductivity $\rho_{o}^{-1}$ and the low-temperature thermopower $S$ are found with the Kubo formalism ${ }^{22}$ as

$$
\rho_{o}^{-1}=\frac{e^{2}}{2 \pi^{2}\left(m^{*}\right)^{2}} \int_{-\infty}^{\infty} d k k^{2}[\operatorname{Im} G(k, \mu)]^{2}
$$

and

$$
S=-\frac{\pi^{2} k_{B}^{2} T}{3 e \epsilon_{0}} \frac{d \ln \rho_{o}}{d \mu} .
$$

If there coexist electronlike and holelike polarons, Eq. (6) must be multiplied by a factor $f_{S}$ with $\left|f_{S}\right| \leqslant 1$. We would like to mention that Eq. (5) should be underestimated due to the fact that the bands are not strictly one-dimensional and that the transport relaxation rate is generally smaller than $\epsilon_{0}$. While the scattering rate and the transport relaxation rate are equal in the noncrossing approximation, they are different for any finite-range scattering potential due to the vertex corrections. Calculating the integral in Eq. (5) leads to

$$
\rho_{o}^{-1}=\frac{e^{2}}{2 a^{2} \pi\left(m^{*} \epsilon_{0}\right)^{1 / 2}} F(\mu),
$$

where

$$
F(\mu)=\frac{\tilde{\mu}\left[\tilde{\mu}+\left(\tilde{\mu}^{2}+g^{2}\right)^{1 / 2}\right]+g^{2} / 2}{g\left[\left(\tilde{\mu}^{2}+g^{2}\right)\left(\tilde{\mu}+\left(\tilde{\mu}^{2}+g^{2}\right)^{1 / 2}\right)\right]^{1 / 2}},
$$

$\tilde{\mu}=\mu-\operatorname{Re} \sigma(\mu)$, and $g=g(\mu)$.

These expressions allow us to calculate the exponents: $\beta_{C}, \beta_{\rho}$, and $\beta_{S}$. The number of carriers does not depend on the isotope substitution, ${ }^{23}$ so that the chemical potential itself 
depends on the polaron mass with the exponent $d \mu / d \ln m^{*}$ $=-2 N / 3 g$, where $N=\int_{-\infty}^{\mu} g(\epsilon) d \epsilon$ is proportional to the total number of states under the Fermi level. Keeping that in mind we finally obtain

$$
\begin{aligned}
\beta_{C} & =\frac{1}{3}\left(1-\frac{2 N g^{\prime}}{g^{2}}\right), \\
\beta_{\rho} & =\frac{2}{3}\left(1+\frac{N F^{\prime}}{g F}\right),
\end{aligned}
$$

and

$$
\beta_{S}=\frac{1}{3}\left(\frac{2 N\left(F^{\prime} / F-F^{\prime \prime} / F^{\prime}\right)}{g}-1\right) .
$$

The prime and the double primes mean the first and the second derivative with respect to $\mu$. The numerical results for the exponents are shown in Fig. 1 as a function of the Fermi level $E_{F} / \epsilon_{0}$. To compare with the experimentally measured oxygen-isotope exponents one should multiply $\beta_{C}, \beta_{\rho}, \beta_{S}$ by the oxygen-isotope exponent $\alpha_{m *}^{\mathrm{O}}$. Because of the logarithmic dependence on the mass ratio [see Eq. (1)], the magnitude of the exponent $\alpha_{m} *$ is expected to be of the order of unity. At large filling, i.e., $\mu \gg 1$, the theory predicts sizable isotope effects on all thermodynamic and kinetic properties with the exponents $\beta_{C}=1, \beta_{\rho}=2$, and $\beta_{S}=1$. This is precisely what one expects from the simple Born approximation for the impurity scattering. The ratio remains the same for any dimension of the polaron spectrum in this doping regime. Interestingly, if the doping is not so high, $\mu$ $\leqslant 1$, the exponent for the thermopower is very small (see Fig. 1), while the exponent for the residual resistivity remains larger than unity. For $\mu=0.25$ and $\alpha_{m *}^{\mathrm{O}} \simeq \alpha_{m *} \simeq$ -1.1 , we obtain $\beta_{C} \simeq 0.45, \beta_{\rho} \simeq 1.45$, and $\beta_{S} \simeq-0.03$, and

$$
\alpha_{C}^{\mathrm{O}} \simeq-0.5, \quad \alpha_{\rho}^{\mathrm{O}} \simeq-1.6, \quad \alpha_{S}^{\mathrm{O}} \simeq 0.0,
$$

i.e., negligible isotope effect on $S$, and more than 3 times lower isotope effect on $C_{\mathrm{el}}$ than on $\rho_{o}$. Applying the exact density of states in the random potential, ${ }^{21}$ we find even a somewhat lower exponent $\beta_{C}$ for $\mu \leqslant 0$ than that in Fig. 1. All these findings are in sharp contrast with those in ordinary metals where $\alpha_{m *}=0$, and there are no isotope effects on $C_{\mathrm{el}}, \rho_{o}$, and $S$.

The choice of $\mu=0.25$ and $\alpha_{m *}^{\mathrm{O}}=-1.1$ is consistent with the experimental results for $\mathrm{Nd}_{0.67} \mathrm{Sr}_{0.33} \mathrm{MnO}_{3}$. Using the measured bare plasma frequency $\hbar \Omega_{p} \simeq 3.3 \mathrm{eV}$ (Ref. 24) and the total carrier density per cell of 0.33 , we obtain the bare hopping integral $t=t_{p p \sigma}=\hbar^{2} / 2 a^{2} m=0.9 \mathrm{eV}$, which is very close to the value $(0.87 \mathrm{eV})$ calculated for $\mathrm{La}_{2} \mathrm{CuO}_{4}{ }^{25}$ From Eq. (1) and $\alpha_{m *} \simeq \alpha_{m *}^{\mathrm{O}} \simeq-1.1$, we get the effective hopping integral $t^{*}=\hbar^{2} / 2 a^{2} m^{*} \simeq 100 \mathrm{meV}$. By setting $\mu=0.25, t^{*}=100 \mathrm{meV}, \epsilon_{0}=27 \mathrm{meV}$, we calculate $C_{\mathrm{el}} / T$ $=26 \mathrm{~mJ} / \mathrm{mol} \mathrm{K}^{2}$ and $\rho_{o}=185 \mu \Omega \mathrm{cm}$ by using Eq. (4) and Eq. (7), respectively. These values are in remarkably good agreement with the measured values: $C_{\mathrm{el}} / T$ $=25(3) \mathrm{mJ} / \mathrm{mol} \mathrm{K}^{2},{ }^{26}$ and $\rho_{o} \simeq 170 \mu \Omega \mathrm{cm}$ for the best

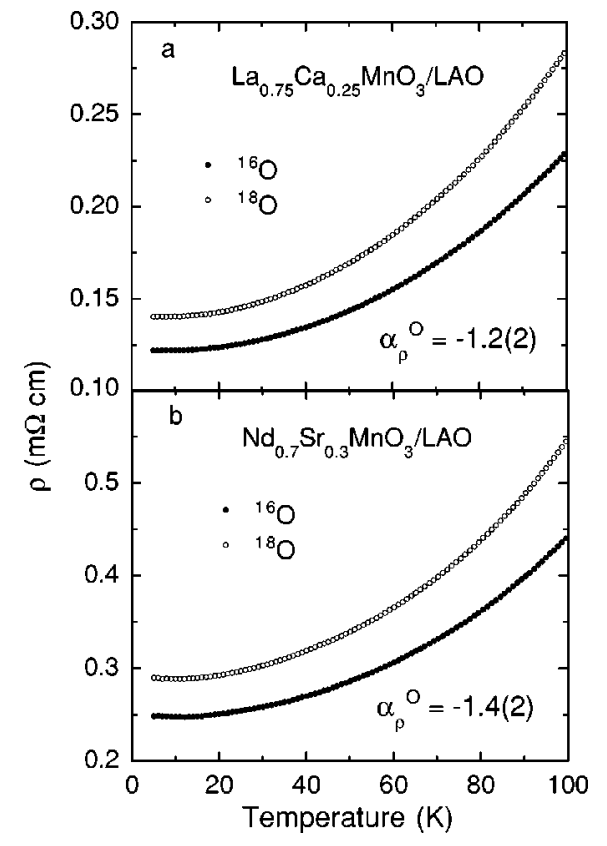

FIG. 2. The low-temperature resistivity of the oxygen-isotope exchanged films of (a) $\mathrm{La}_{0.75} \mathrm{Ca}_{0.25} \mathrm{MnO}_{3}$ and (b) $\mathrm{Nd}_{0.7} \mathrm{Sr}_{0.3} \mathrm{MnO}_{3}$. In both cases, the residual resistivity $\rho_{o}$ for the ${ }^{18} \mathrm{O}$ samples is larger than for the ${ }^{16} \mathrm{O}$ samples by $15 \%$ (Ref. 23) The oxygenisotope exponent $\alpha_{\rho}^{\mathrm{O}}$ was calculated from the definition: $\alpha_{\rho}^{\mathrm{O}}=$ $-d \ln \rho_{o} / d \ln M_{\mathrm{O}}$ ( $M_{\mathrm{O}}$ is the oxygen mass).

single crystals. ${ }^{27,28}$ Furthermore, the value of $\epsilon_{0}$ is in good agreement with the measured transport relaxation rate $(\sim 24 \mathrm{meV})$ for $\mathrm{La}_{0.7} \mathrm{Ca}_{0.3} \mathrm{MnO}_{3} .{ }^{29}$ This justifies the choice of $\mu=0.25$ and $\alpha_{m *}^{\mathrm{O}}=-1.1$ for $\mathrm{Nd}_{0.67} \mathrm{Sr}_{0.33} \mathrm{MnO}_{3}$. For $\mathrm{La}_{0.75} \mathrm{Ca}_{0.25} \mathrm{MnO}_{3}$, we calculate $C_{\mathrm{el}} / T=7.5 \mathrm{~mJ} / \mathrm{mol} \mathrm{K}{ }^{2}$ and $\rho_{o}=150 \mu \Omega \mathrm{cm}$ by setting $\mu=0.70$ and $\alpha_{m *}^{\mathrm{O}}=-0.7$. The measured $\rho_{o}$ for $\mathrm{La}_{0.75} \mathrm{Ca}_{0.25} \mathrm{MnO}_{3}$ is $120-150 \mu \Omega \mathrm{cm}$ [see Fig. 2(a) and discussion in Ref. 23]. From our specific-heat data for $\mathrm{La}_{0.75} \mathrm{Ca}_{0.25} \mathrm{MnO}_{3}$, we estimate $C_{\mathrm{el}} / T$ $\simeq 7 \mathrm{~mJ} / \mathrm{mol} \mathrm{K}^{2}$, which is slightly lower than the one $\left(\sim 8 \mathrm{~mJ} / \mathrm{mol} \mathrm{K}{ }^{2}\right)$ for $\mathrm{La}_{0.8} \mathrm{Ca}_{0.2} \mathrm{MnO}_{3} \cdot{ }^{30}$ With $\mu=0.70$ and $\alpha_{m *}^{\mathrm{O}}=-0.7$, we have

$$
\alpha_{C}^{\mathrm{O}} \simeq-0.5, \quad \alpha_{\rho}^{\mathrm{O}} \simeq-1.2, \quad \alpha_{S}^{\mathrm{O}} \simeq-0.17 .
$$

Experimentally, the oxygen-isotope effect on the intrinsic low-temperature resistivity has been studied ${ }^{23}$ in high-quality thin films of $\mathrm{La}_{0.75} \mathrm{Ca}_{0.25} \mathrm{MnO}_{3}$ and $\mathrm{Nd}_{0.7} \mathrm{Sr}_{0.3} \mathrm{MnO}_{3}$. Our thin films were thick $(2000 \AA)$, and were annealed at $940^{\circ} \mathrm{C}$ for $10 \mathrm{~h}$, so no strains could remain in the films. We did backexchange, and we knew precisely the oxygen-isotope enrichment (more details can be found in Refs. 23 and 31). In Fig. 2 we plot the low-temperature resistivity of the oxygenisotope exchanged films of (a) $\mathrm{La}_{0.75} \mathrm{Ca}_{0.25} \mathrm{MnO}_{3}$ and (b) $\mathrm{Nd}_{0.7} \mathrm{Sr}_{0.3} \mathrm{MnO}_{3}$. In both cases, the residual resistivity $\rho_{o}$ for the ${ }^{18} \mathrm{O}$ samples is larger than for the ${ }^{16} \mathrm{O}$ samples by about $15 \%$. The observed large oxygen-isotope effect on $\rho_{o}$ is consistent with the above theoretical prediction for polaronic metals. From the definition of the oxygen-isotope exponent: $\alpha_{\rho}^{\mathrm{O}}=-d \ln \rho_{o} / d \ln M_{\mathrm{O}}\left(M_{\mathrm{O}}\right.$ is the oxygen mass), we obtain 
$\alpha_{\rho}^{\mathrm{O}}=-1.4(2)$ for $\mathrm{Nd}_{0.7} \mathrm{Sr}_{0.3} \mathrm{MnO}_{3}$, and $\alpha_{\rho}^{\mathrm{O}}=-1.2(2)$ for $\mathrm{La}_{0.75} \mathrm{Ca}_{0.25} \mathrm{MnO}_{3}$. These values are in excellent agreement with Eq. (12) and Eq. (13), respectively.

The quantitative agreement between the resistivity data and the theory indicates that the low-temperature metallic state in doped manganites is a polaronic Fermi liquid. To further confirm this, one should experimentally demonstrate that $\alpha_{S}^{\mathrm{O}}$ is negligible and that $\alpha_{C}^{\mathrm{O}}$ is rather small in these materials. Our preliminary results of the oxygen-isotope effect on the low-temperature specific heat in $\mathrm{La}_{1-x} \mathrm{Ca}_{x} \mathrm{MnO}_{3}$ are consistent with Eq. (12) within the experimental uncertainty. Here we show the data of the thermopower $S$ at low temperatures for the isotope-exchanged $\mathrm{La}_{1-x} \mathrm{Ca}_{x} \mathrm{MnO}_{3}$. The detailed measurement technique was described in Ref. 32. Figure 3 shows the thermopower $S$ below $60 \mathrm{~K}$ for the ${ }^{16} \mathrm{O}$ and ${ }^{18} \mathrm{O}$ samples of $\mathrm{La}_{1-x} \mathrm{Ca}_{x} \mathrm{MnO}_{3}$ with $x=0.20$ and 0.25 . The isotope-exchanged samples are the same as those reported in Ref. 31. It is apparent that the oxygen-isotope effect on $S$ is negligible below $20 \mathrm{~K}$ for both compositions $\left(\alpha_{S}^{\mathrm{O}} \simeq 0.0\right)$. The negligible $\alpha_{S}^{\mathrm{O}}$ and the substantial $\alpha_{\rho}^{\mathrm{O}} \sim$ -1.3 observed in these samples are in quantitative agreement with the theoretical prediction [Eq. (12) and Eq. (13)]. Therefore, the present theoretical and experimental studies of

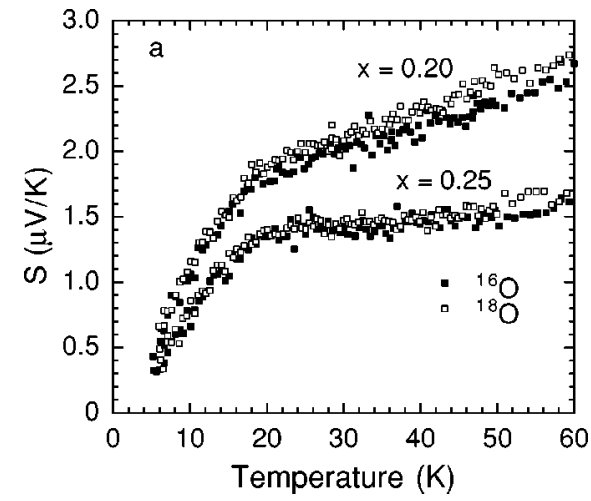

FIG. 3. The temperature dependence of the thermopower $S$ of the ${ }^{16} \mathrm{O}$ and ${ }^{18} \mathrm{O}$ samples of $\mathrm{La}_{1-x} \mathrm{Ca}_{x} \mathrm{MnO}_{3}$ below $60 \mathrm{~K}$.

the low-temperature kinetic and thermodynamic properties provide strong evidence for the existence of polaronic Fermi liquid in manganites.

We would like to thank K. A. Müller for valuable comments. The work at Zürich was supported by the Swiss National Science Foundation. The work at Houston was supported in part by the U.S. National Science Foundation, and work at Berkeley by the U.S. Department of Energy.
${ }^{1}$ L.D. Landau, J. Phys. (USSR) 3, 664 (1933).

${ }^{2}$ A. S. Alexandrov and N. F. Mott, Polarons and Bipolarons (World Scientific, Singapore, 1995).

${ }^{3}$ J. T. Devreese, Encyclopedia of Applied Physics (VCH Publishers, New York, 1996), Vol. 14, p. 383.

${ }^{4}$ A.S. Alexandrov, Phys. Rev. B 61, 12315 (2000).

${ }^{5}$ A.S. Alexandrov, Phys. Rev. B 46, 14932 (1992).

${ }^{6}$ J. Bonca, T. Katrasnik, and S. Trugman, Phys. Rev. Lett. 84, 3153 (2000).

${ }^{7}$ G.M. Zhao and D.E. Morris, Phys. Rev. B 51, R16 487 (1995).

${ }^{8}$ G.M. Zhao et al., Nature (London) 385, 236 (1997).

${ }^{9}$ J. Hofer et al., Phys. Rev. Lett. 84, 4192 (2000).

${ }^{10}$ M. Jaime et al., Phys. Rev. Lett. 78, 951 (1997).

${ }^{11}$ G.M. Zhao et al., Nature (London) 381, 676 (1996).

${ }^{12}$ A. Shengelaya et al., Phys. Rev. B 61, 5888 (2000).

${ }^{13}$ A.S. Alexandrov and A.M. Bratkovsky, Phys. Rev. Lett. 82, 141 (1999).

${ }^{14}$ For a recent review, see Y. Tokura and N. Nagaosa, Science 288, 462 (2000).

${ }^{15}$ J.S. Zhou and J.B. Goodenough, Phys. Rev. Lett. 80, 2665 (1998).
${ }^{16}$ M. Uehara et al., Nature (London) 399, 560 (1999).

${ }^{17}$ I.G. Lang and Yu.A. Firsov, Sov. Phys. JETP 16, 1301 (1963).

${ }^{18}$ T. Saitoh et al., Phys. Rev. B 51, 13942 (1995).

${ }^{19}$ H.L. Ju, H.C. Sohn, and K.M. Krishnan, Phys. Rev. Lett. 79, 3230 (1997).

${ }^{20}$ P.E. Kornilovitch, Phys. Rev. B 59, 13531 (1999).

${ }^{21}$ H.L. Frisch and S.P. Lloyd, Phys. Rev. 120, 1175 (1960).

${ }^{22}$ R. Kubo, H. Hasegava, and N.J. Hashitsume, J. Phys. Soc. Jpn. 14, 56 (1959).

${ }^{23}$ G.M. Zhao et al., cond-mat/0008029 (unpublished).

${ }^{24}$ Guo-meng Zhao et al., Phys. Rev. Lett. 84, 6086 (2000).

${ }^{25}$ A.K. McMahan, R.M. Martin, and S. Satpathy, Phys. Rev. B 38, 6650 (1988).

${ }^{26}$ J.E. Gordon et al., Phys. Rev. B 59, 127 (1999).

${ }^{27}$ P. Dai et al., Phys. Rev. B 61, 9553 (2000).

${ }^{28}$ Y. Sawaki et al., Phys. Rev. B 61, 11588 (2000).

${ }^{29}$ J.R. Simpson et al., Phys. Rev. B 60, R16 263 (1999).

${ }^{30}$ J.J. Hamilton et al., Phys. Rev. B 54, 14926 (1996).

${ }^{31}$ Guo-meng Zhao et al., Phys. Rev. B 60, 11914 (1999).

${ }^{32}$ A.K. Heilman et al., Phys. Rev. B 61, 8950 (1999). 